\title{
Philosophiques
}

\section{Le réel, inconnu à cette adresse}

\section{Aude Bandini}

Volume 45, numéro 1, printemps 2018

URI : https://id.erudit.org/iderudit/1048625ar

DOI : https://doi.org/10.7202/1048625ar

Aller au sommaire du numéro

Éditeur(s)

Société de philosophie du Québec

ISSN

0316-2923 (imprimé)

1492-1391 (numérique)

Découvrir la revue

Citer ce document

Bandini, A. (2018). Le réel, inconnu à cette adresse. Philosophiques, 45(1),

249-260. https://doi.org/10.7202/1048625ar

Ce document est protégé par la loi sur le droit d'auteur. L'utilisation des services d'Érudit (y compris la reproduction) est assujettie à sa politique d'utilisation que vous pouvez consulter en ligne.

https://apropos.erudit.org/fr/usagers/politique-dutilisation/ 


\title{
Le réel, inconnu à cette adresse
}

\author{
AUDE BANDINI \\ Département de philosophie \\ Université de Montréal
}

Avec L'adresse du réel, Jocelyn Benoist poursuit une réflexion originale sur le réalisme entreprise depuis au moins les années 2000 , et à laquelle il avait déjà notamment consacré ses précieux Éléments de philosophie réaliste ${ }^{1}$. Ce nouvel opus revient avec attention sur ce que cela signifie fondamentalement que d'être réaliste en philosophie, si tant est que le «réalisme» ne soit pas qu'une entité purement nominale, renvoyant à des doctrines différentes et parfois même opposées.

À cet égard, il semble bien que Benoist partage avec Putnam² l'idée que, quelle que soit la forme sous laquelle il se présente, le réalisme "métaphysique » échoue nécessairement à tenir ses promesses, notamment celle de pouvoir une fois pour toutes sauver l'innocente Princesse du Sens commun, face à ses ennemis héréditaires (l'idéalisme, le kantisme et le néo-kantisme, le pragmatisme ou encore l'anti-réalisme). Ce qu'il a en tête dans L'adresse $d u$ réel, c'est le type de réalisme épistémologique et métaphysique qui dérive de la philosophie kantienne, magistralement endossé au vingtième siècle par des auteurs tels que C. I. Lewis, Wilfrid Sellars ou encore John McDowell, et que les partisans du "Nouveau Réalisme», par exemple Maurizio Ferraris, Markus Gabriel ou Quentin Meillassoux, entendent dépasser. Les raisons pour lesquelles Benoist demeure réservé quant à la question de savoir s'ils y parviennent mériteraient qu'on s'y attarde davantage. Faute d'espace, je me concentrerai néanmoins plus directement sur ce qui, selon Benoist, pourrait constituer un réalisme bien compris en philosophie, c'est-à-dire, et en dépit des apparences de paradoxe, un "réalisme sans métaphysique».

L'entreprise de Jocelyn Benoist, telle qu'il la présente dans le premier chapitre de l'ouvrage qui nous intéresse, consiste à "essayer d'isoler, pour autant que cela ait un sens, un trait général de l'orchestration [qu'il propose de qualifier] de "métaphysique" du thème réaliste ${ }^{3}$ ", En s'appuyant sur la critique du "corrélationisme » développée par Meillassoux, Benoist dénonce comme absurde toute métaphysique et toute théorie de la connaissance qui impliqueraient de séparer les choses telles qu'elles nous apparaissent des choses telles qu'elles sont en soi, avec l'idée que nos facultés cognitives (ou la pensée en général) ne nous permettraient jamais d'atteindre que les premières, sans jamais nous dissuader de croire en l'existence des secondes, ni

1. Jocelyn Benoist, Éléments de philosophie réaliste, Paris, Vrin, 20 I I.

2. Hilary Putnam, The Many Faces of Realism, LaSalle, Open Court, I987.

3. Jocelyn Benoist, L'adresse du réel, Paris, Vrin, 20I7, p. I7-I8. 
par conséquent nous décourager de chercher à découvrir un moyen d'y accéder dans leur supposée "pureté». La métaphysique à laquelle il s'attaque comme à un mythe est ainsi celle qui pose, d'une part, l'existence d'une pensée capable de transcender ses propres limites (se voulant ainsi "affranchie de toute condition de pensée $\left.{ }^{4} »\right)$, et d'autre part et du même coup, l'existence d'un «être pur», qui cesserait immédiatement d'être luimême à partir du moment où il serait pensé. Là contre, l'objectif de Benoist consiste à défendre la thèse selon laquelle:

l'être est au contraire précisément ce qui est saisi par nos pensées, c'est-à-dire ce qui est normé par elles, telles que nous les entretenons en des circonstances réelles, dans lesquelles nous sommes confrontés à la réalité même et où celle-ci revêt une physionomie concrète et déterminable. L'être spéculatif, censé posséder son sens (ou non-sens) univoque au-delà de ces déterminations que leur contextualité ancre dans la réalité, n'est rien d'autre qu'une idole métaphysique, négatif photographique d'une pensée oublieuse de ses limites c'est-à-dire de cette même réalité.

Tel est le réalisme que je voudrais défendre dans ce livres.

La clef, si l'on veut s'extraire d'une telle métaphysique, fondamentalement absurde parce que, par principe, elle exclut la possibilité d'être autre chose que parfaitement vaine, consiste selon Benoist en un contextualisme épistémologique et un réalisme qu'on pourrait dire «déflationniste» sur le plan métaphysique, dont il a déjà avancé une première caractérisation dans ses Éléments de philosophie réaliste. À l'aide de ces deux outils théoriques, et pour reprendre les termes de la petite histoire racontée par Putnam, le Chevalier réaliste pourrait parvenir à honorer les termes de son contrat de mariage avec la Princesse du Sens commun, au lieu de finir par lui annoncer un beau matin et au milieu du petit déjeuner, qu'elle n'obtiendra finalement rien de ce qui lui avait été promis au moment de leurs vœux (à savoir des choses réelles, et pas seulement des choses «pensées »).

Ainsi, le réalisme que défend Benoist entend donner droit à nos intuitions ordinaires concernant ce qui est réel ou objectif et ce qui ne l'est pas, à la distinction que nous faisons habituellement entre les mots et les choses, le vrai et le faux, le naturel et le social, les normes et le monde. Avant de nous pencher sur la question de savoir s'il y parvient (la Princesse a-t-elle encore été flouée?), tâchons d'en présenter les principaux traits et attraits.

Ce qui est peut-être symptomatique de la contamination du réalisme ordinaire par une encombrante métaphysique - laquelle crée en vérité plus de problèmes qu'elle n'en résout - est la représentation relationaliste ou distale du rapport entre l'esprit et le monde sur laquelle elle débouche, et que Benoist critique sous le nom de «discours de l'accès ${ }^{6}$ : l'idée serait ici
4. Ibid., p. 22.
5. Ibid.
6. Ibid., p. 20. 
que la pensée (comme le langage, du moins dans ses usages descriptifs) aurait pour tâche de rejoindre une réalité toujours extérieure et à distance du sujet, ou d'y accéder. C'est la présence de cette distance qui permettrait d'expliquer des phénomènes épistémologiquement cruciaux, comme la distinction entre les croyances vraies et les croyances fausses, les illusions ou les apparences et la réalité. Le problème est qu'une fois que l'on a installé cette distance, on se trouve bien en peine d'expliquer comment elle peut être franchie, et même si elle peut tout simplement l'être.

L'une des motivations du réalisme ordinaire, remarque Benoist, se trouve dans la volonté de rendre compte de l'intuition du sens commun selon laquelle il existe bien quelque chose comme le réel "objectif ", qui est ce qu'il est, quoi que nous en pensions ou nous en disions. Ce qui est réel se définit alors par son "indépendance " relativement à l'esprit ou aux esprits. Selon Benoist, il est cependant possible de rendre compte de cette intuition réaliste sans pour autant souscrire au «discours de l'accès » et à la métaphysique qui l'accompagne. C'est du moins ce qu'il s'efforce de mettre en évidence à l'occasion d'une discussion serrée concernant «l'objectivité de la vérité». Ce dont il faut sauver la Princesse du Sens commun dans ce cadre, c'est le relativisme épistémologique. Celui-ci peut être écarté, soutient Benoist, à partir du moment où l'on reconnaît que la grammaire même de notre usage du prédicat de vérité nous engage à admettre, c'est-à-dire penser et agir en partant du principe que l'énoncé que nous tenons pour vrai a cette valeur, indépendamment de toute capacité humaine à le saisir ou non comme tel. Cette approche de la vérité est déflationniste, au sens où elle est métaphysiquement neutre ou même nulle: il ne s'agit pas de poser une réalité absolue et plus ou moins cachée qui attendrait qu'on la découvre, car ce serait là se commettre au mythe métaphysique de l'être "pur» et intangible qu'il s'agit justement de dénoncer.

Mais il ne s'agit pas non plus de réduire la vérité au caractère assertable ou non d'un énoncé dans un cadre linguistique ou conceptuel donné et de nature strictement conventionnelle ou arbitraire. Il existe selon Benoist une voie qui nous permet d'éviter Charybde (l'absolutisme) sans tomber sur Scylla (le relativisme). Cette voie est justement celle, étroite et parfois tortueuse, que le contextualisme permettrait de rendre navigable. Qu'une telle voie existe est loin d'être évident, dans la mesure où celle-ci consisterait à offrir au réalisme quelque chose qui aurait finalement l'autorité normative d'une transcendance - un être qui ne serait pas seulement un être connu, et dont on pourrait dire des choses vraies ou fausses - sans quitter l'espace de l'immanence. Le risque est alors grand, comme nous le verrons, de n'échapper aux sirènes du relativisme autrement qu'en se ligotant au mât de l'idéalisme, notamment dans sa version linguistique.

Avant d'en arriver à ce point, il nous faut dire quelques mots de ce en quoi consiste ce contextualisme, sans lequel le réalisme ne semble pas pouvoir tenir ses promesses. Selon Benoist, il vise à rendre compte du fait que 
«si le fait que nos vérités [...] soient vraies n'est nullement relatif à nous ce serait contraire à la grammaire du mot "vrai" — le format de ces vérités, lui, l'est fondamentalement ${ }^{7}$ ». Il ne s'agit donc pas d'affirmer, comme semble le faire Bruno Latour, que la vérité de l'énoncé «Ramsès II est mort de la tuberculose» dépend du contexte, et que si cet énoncé est vrai aujourd'hui (quoi que le point ne soit pas tout à fait clair), il ne l'était pas avant que le bacille de Koch eut été découvert. L'idée est plutôt que le contenu sémantique que nous attribuons à cet énoncé est lui-même déterminé par des facteurs contextuels, comme l'est en fait le contenu de toute pensée. Tel que Benoist le conçoit,

le contextualisme, loin d'être, comme le relativisme, un antiréalisme, se définit au contraire comme une tentative de mettre en évidence l'ancrage réel sans lequel la pensée se réduit à une ombre: un réalisme, donc, non seulement de l'objet de pensée, mais eu égard aux formats réels de la pensée - selon lesquels seulement il peut y avoir "objet".

Une des difficultés auxquelles le lecteur peut se trouver confronté, lorsqu'il s'agit de saisir correctement ce que Benoist entend par «contextualisme » (et par conséquent par "réalisme ») dans cet ouvrage, tient à ce que l'usage qu'il fait de ce concept est mené de manière assez indépendante des discussions qui se tiennent sur le contextualisme en épistémologie contemporaine, notamment outre-Atlantique. À la différence de ce contextualisme que l'on pourrait dire "académique ", celui que défend Benoist est très large et ne concerne pas que les énoncés méta-épistémologiques, dans lesquels nous attribuons ou nous auto-attribuons des connaissances, ou des justifications. Plus près des philosophes du langage ordinaire, notamment Austin et surtout le Wittgenstein des Investigations philosophiques, il considère que tout contenu propositionnel est justiciable d'une analyse en termes d'usage, et ce faisant de contexte: il n'y a tout simplement "pas de signification sans contextualité; mais aussi, inversement, pas de contextualité sans signification $^{9}{ }^{\prime}$. Il s'agit donc d'abord d'un contextualisme sémantique ou d'une théorie contextualiste de la signification, mais dont l'application spécifique dans le champ épistémologique est naturellement décisive pour toute réflexion ontologique. C'est pourquoi il est effectivement tout à fait nécessaire de s'intéresser, comme le fait Benoist, à la grammaire d'un prédicat tel que «vrai », puisque c'est bien celui-ci qui permet d'effectuer l'articulation entre la réflexion sémantique et la réflexion ontologique.

À cet égard, l'originalité de la thèse défendue dans L'adresse du réel tient au fait que ce sont essentiellement les rapports variés que nous entretenons au monde, qu'ils soient perceptuels ou intellectuels, et ce faisant «la

7. Ibid., p. 26.

8. Ibid., p. 27.

9. Benoist, Éléments de philosophie réaliste, p. 77-78. 
réalité des attitudes que nous adoptons par rapport à lui et [1]es types d'attentes normatives que nous avons réellement à son endroit ${ }^{10}$ » qui déterminent non pas ce qui est vrai en soi, mais ce dont la vérité nous importe. À l'instar de Wittgenstein, mais aussi de James, il faut effectivement reconnaître que certaines hypothèses sont "mortes ", "évitables» ou encore «insignifiantes» pour nous, précisément en raison du contexte qui nous conduit à nous poser (ou non) la question de l'existence de certaines choses, de la signification ou de la vérité de certains énoncés: ce contexte-là est bien réel, et effectivement source de régulation normative.

Le risque que ce contextualisme, notamment dans sa déclinaison épistémologique, soit pris à tort pour une forme de relativisme peut alors, selon Benoist, être évité à partir du moment où l'on conçoit correctement le type de dépendance logique qui unit la réalité et la vérité, et qui est bien celui que pose le réalisme: la vérité présuppose la réalité, et non l'inverse. Faute d'être pourvus d'un entendement archétypique, nous ne donnons certainement pas l'être à nos êtres de langage. Cependant, pour l'apercevoir il faut se méfier sinon de la grammaire de surface des énoncés, du moins de nos tendances à nous croire ontologiquement engagés là où nous ne le sommes nullement, si cela veut dire poser des choses hors et à distance de l'esprit. Notamment, le fait de reconnaître qu'il y a bien ce que Benoist appelle un «enracinement ontologique de la vérité ${ }^{11}$ » n'implique nullement que le fait d'entretenir des croyances vraies signifierait qu'on serait parvenu par-là à saisir un être «pur» et mythique tel que celui que poursuit en vain le métaphysicien réaliste ordinaire. Un réalisme "sans la métaphysique» est précisément celui qui s'efforce de rendre compte de la dépendance logique de la vérité et, plus largement, de nos énoncés et de nos pensées (en vertu de quoi ceux-ci peuvent parfois être faux) face à la réalité, sans faire de cette dernière une sorte d'entité ou de système d'entités, subsistant dans un arrière-monde séparé, inaltérable et toujours identique à lui-même, mais auquel, comme se plaît à le souligner le sceptique, nous ne sommes jamais bien sûrs d'avoir accès.

Reste alors à savoir comment, par ce contextualisme, nous pourrions bien conserver les avantages de la transcendance (sa force normative) sans ses inconvénients (le discours de l'accès, et sa contrepartie métaphysique). C'est sur ce point que se déploie ce qui constitue peut-être le plus grand morceau de bravoure de cet ouvrage, à savoir une théorie de la signification résolument internaliste et qui envisage le langage, ou plutôt nos actes de langage, comme ce qui, parce qu'ils sont eux-mêmes réels et constituent des faits du monde, font advenir la réalité tout simplement parce qu'ils la présupposent. Cette sémantique se trouve présentée au chapitre IV, dédié à la notion wittgensteinienne de grammaire, particulièrement bienvenue dans la

10. Benoist, L'adresse du réel, p. 28.

11. Ibid., p. 38 . 
mesure où le lecteur aurait pu avoir jusque-là l'impression que le réalisme de Benoist n'avait pas réellement les moyens de ses ambitions, et notamment, que la Princesse du Sens commun à laquelle il semblait avoir promis, entre autres bienfaits, la confirmation de la distinction entre l'ordre du langage et celui des choses, en serait encore pour ses frais.

Plus précisément, on pourrait se demander dans quelle mesure il parvient, comme il le prétend, à préserver «l'objectivité de la vérité », puisque la théorie de la vérité sur laquelle il s'appuie ne nous explique pas ce qui rend vrais les énoncés vrais. Elle est déflationniste, on l'a vu, au sens où elle se fonde sur la grammaire du prédicat «vrai» tel qu'il intervient dans nos énoncés, et les rôles qu'il est susceptible de jouer dans nos différents jeux de langage. Il semble qu'il n'y aurait rien d'autre à dire sur la vérité, sauf à retomber dans le type de métaphysique qu'il s'agit justement de courtcircuiter. Le commentaire que Benoist offre d'Aristote ${ }^{12}$ est particulièrement frappant à cet égard, puisqu'il fait, de manière pour le moins hétérodoxe mais parfaitement convaincante, d'un énoncé qui a toujours été interprété en un sens métaphysique, une remarque grammaticale au sujet du prédicat «vrai ». Le problème est que l'on peut se demander alors si ce qui nous est proposé est bien une théorie de la vérité, susceptible de fonder effectivement un réalisme contextualiste, ou bien seulement une théorie $d u$ concept de vérité ou de l'usage de ce concept, de laquelle on ne pourrait tirer aucune conclusion épistémologique ni ontologique qui vaille. Faute d'une explication concernant la manière dont le langage se rapporte au monde, la différence entre la vérité et la fausseté, la connaissance et l'erreur, on peut se demander quel intérêt il reste encore à être réaliste en quel que sens que ce soit - navré, Princesse.

L'objection est celle, bien connue mais rarement bien surmontée par les théoriciens de la signification comme usage, de l'«idéalisme linguistique». Celle-ci, avance Benoist, "indique avant tout une méprise sur la nature du langage ${ }^{13}$ ", méprise qui consiste à réduire le langage à sa fonction descriptive ou représentationnelle, sans s'apercevoir, d'une part, que celle-ci n'est qu'une partie de tout ce que l'on peut faire avec des mots, et d'autre part, que le phénomène de la signification linguistique n'est pas de l'ordre d'une relation qui s'établirait entre des entités purement linguistiques, des mots ou des énoncés, et de "vraies" choses. Comme le remarque Benoist, il est à peu près incontestable que nos actes de langage, comme de manière générale tout phénomène social, sont bien, eux aussi, de "vraies choses ", c'est-à-dire des éléments du réel, susceptibles d'entretenir des relations causales avec d'autres objets, individus ou faits: selon les circonstances, je peux me faire ouvrir la porte en donnant le mot de passe, endormir un amphithéâtre d'étudiants au complet en professant un cours sur Sellars, ou encore

12. Ibid., p. 36-37.

13. Ibid., p. I 26. 
faire rentrer le chat en l'appelant par la fenêtre. Sellars, puisque nous venons de l'évoquer, insistait sur la double nature des énoncés (comme des pensées), comme phénomènes normativement déterminés, mais aussi comme "objets naturels linguistiques", relevant ainsi aussi bien de l'espace logique des raisons que de celui, naturel des causes ${ }^{14}$. Le point crucial ici est que le fait de représenter, référer, dénoter, signifier ou encore vouloir dire, ne renvoie jamais à rien d'autre qu'à des types d'usages, réels, du langage. Le reconnaître, c'est, conformément à ce que souhaitait Benoist, nous ramener dans le champ de l'immanence.

Reste alors à rendre compte de l'ordre de la normativité, ce en vertu de quoi certains énoncés sont doués ou bien dépourvus de signification, vrais ou bien faux, de même que certains actes sont, selon les circonstances, appropriés ou non: quoi qu'on puisse en penser par ailleurs, on ne fait pas plus de révérence pour saluer sa concierge qu'on ne donne une tape dans le dos à la reine d'Angleterre. C'est alors qu'intervient, à nouveau, la ressource théorique du contextualisme, avec l'idée, d'une part, que les mots ne fonctionnent comme tels et ne peuvent acquérir de sens que dans un contexte, jeu de langage ou forme de vie (et non pas de manière atomique ou isolée de toute pratique), et d'autre part, que saisir ce que signifie un énoncé ne renvoie pas à en connaître les conditions de vérité, mais plutôt à maîtriser les règles qui, dans les circonstances, président à son usage. Et c'est ainsi la maîtrise pratique de l'usage d'un énoncé qui permet d'en établir, si on le souhaite, les conditions de vérité. Pas l'inverse.

Ce qui se fait alors jour, c'est que le réel est toujours premier, y compris dans et par le langage: il est, pour reprendre les termes des Éléments de philosophie réaliste, ce que l'on a déjà, et non ce qu'il faudrait chercher parce qu'il serait toujours à distance et plus ou moins caché de nous. Les représentations ou les apparences ne sont donc pas ce dont nous partons, et qu'il faudrait dépasser pour accéder au réel: elles en sont au contraire dérivées. Il s'agit là aussi d'un point de grammaire, également aperçu par Sellars, selon lequel les modes de discours de l'apparaître constituent toujours une forme parasite du mode de discours du réel ${ }^{15}$. Si j'affirme que, dans la figure de Müller-Lyer, l'un des segments est plus grand que l'autre, je réalise un acte de langage réel, qui a des conséquences réelles, comme le fait que l'on me contredira peut-être, ou que, si je mesure par moi-même la longueur des deux segments, je serai surpris par le résultat. Si, pour paraphraser Thimothy Williamson, on considère que "le réel vient en premier ", il n'est pertinent d'introduire l'usage du vocabulaire des apparences ou de l'illusion qu'à partir du moment où il permet de rendre compte d'une différence dans

14. Wilfrid Sellars, Naturalism and Ontology, Atascadero, Ridgeview Publishing Company, I979.

15. Wilfrid Sellars, Empirisme et philosophie de l'esprit, traduction de Fabien Cayla, Paris, Éditions de l'Éclat, I992, p. 36-49. 
le réel, c'est-à-dire dans l'usage. On retrouve ici l'argument pragmatiste familier, selon lequel être, c'est faire une différence. Que les segments de droite qui constituent la figure que je regarde soient d'égale longueur ou non, cela fait bien une différence, peut-être pas du seul point de vue de ce que je perçois ici et maintenant, mais du point de vue de tout ce à quoi cette perception est normativement reliée, par exemple si je décide de réaliser une mesure. Par contraste, la thèse sceptique radicale selon laquelle il se pourrait que tout ce que nous croyons réel ne soit qu'illusion et que nous ne soyons que des cerveaux dans des cuves, ou encore que le monde a été créé il y a seulement cinq minutes, avec tout ce qu'il contient, y compris nos souvenirs, les fossiles et les vestiges de l'Antiquité, ces thèses-ci n'ont aucune pertinence: qu'elles soient vraies ou fausses ne fait finalement aucune différence dans le réel, entendu une fois encore comme ce que l'on a et le type d'engagements normatifs qui sont les nôtres. Quand bien même j'admettrais que le monde vient à peine d'être créé, il me faudra encore payer mes impôts.

La charge de la preuve épistémologique ou métaphysique n'incombe donc pas à celui qui tient le réel pour acquis, mais au contraire à celui qui estime qu'on devrait le conquérir et qu'il faut pour cela s'être d'abord assuré qu'il pouvait l'être, en dépit des limites de notre pouvoir de connaître. En ce sens, on pourrait considérer que la Princesse du Sens commun, dans son union avec le Réalisme (sans métaphysique), reçoit bien ce qui lui avait été promis, et que l'idéalisme, le relativisme, ou encore le corrélationisme menaçaient de lui ravir. Elle peut désormais leur répondre: «Messieurs les métaphysiciens, tirez les premiers.»

On pourrait cependant s'inquiéter de savoir, en définitive, ce qui reste de l'adresse du réel pris en ce sens qu'on pourrait dire strictement transcendantal (et non transcendant), dans la mesure où il est à lui-même sa condition de possibilité, ou du moins de la possibilité de toute interrogation, enquête ou énoncé sur ce qu'il est. S'il est aussi contextuellement déterminé et relatif à des usages (linguistiques mais pas uniquement) que Benoist le suggère, on pourrait craindre qu'il ne soit rien d'autre qu'une fonction grammaticale dont l'argument ne serait jamais saturé, ou plutôt, qui pourrait être saturé par n'importe quoi. Autrement dit, le réel n'aurait pas d'essence propre et serait, pour faire écho au titre de l'ouvrage, sans domicile fixe. En un certain sens, on pourrait même considérer que prétendre avoir fixé, une fois pour toutes, l'adresse du réel est en fait toujours le signe que l'on a cédé à la tentation et que, pour paraphraser Kant, on est - encore - revenu à la métaphysique comme à la maîtresse avec laquelle on s'était brouillé. L'argumentation de Jocelyn Benoist implique alors l'adoption d'une position particulièrement radicale à l'égard de la métaphysique comme discipline censée nous éclairer sur "ce qu'il y a " dans le monde, au sens le plus général du terme, et dont on pourrait s'étonner qu'elle ne soit pas plus directement thématisée comme telle. Le simple fait que l'ouvrage s'ouvre sur un chapitre intitulé «Le réalisme sans la métaphysique » suffit cependant à 
signaler que cette réflexion, de nature métaphilosophique, est certainement déjà à l'œuvre dans la pensée de ce philosophe.

Nous proposons donc, pour finir, de déterminer ce en quoi cette métaphilosophie pourrait consister. Pour cela, il nous semble pertinent de nous appuyer sur un type de projet philosophique qui, on le verra, entretient des similitudes frappantes avec celui de Jocelyn Benoist, et dont l'orientation générale est également fortement marquée par la philosophie de Wittgenstein (dans De la certitude, plus encore que dans Investigations): le contextualisme de Michael Williams. Nous avons déjà souligné que le contextualisme défendu par Benoist relevait d'abord de la sémantique, et qu'il se distinguait du contextualisme aujourd'hui débattu par un certain nombre de théoriciens de la connaissance par le fait qu'il ne s'intéressait pas, ou du moins pas prioritairement, aux énoncés d'attribution de connaissance. On remarquera notamment que si Benoist s'intéresse à la grammaire de la vérité, il ne réserve aucun traitement spécifique à celle de la justification. Il n'y a cependant rien, dans l'appareil théorique qu'il mobilise, qui s'oppose à ce qu'une analyse de ce genre soit conduite - elle l'est peut-être même, virtuellement, déjà.

Si nous choisissons de nous intéresser aux écrits de Michael Williams, c'est précisément parce qu'il a avancé une thèse de ce genre, dans le cadre d'une réflexion extrêmement pénétrante sur le problème du scepticisme en épistémologie, et qui vise à montrer que la menace sceptique, si elle existe effectivement, n'est en réalité que l'avatar du projet épistémologique luimême, tel du moins qu'il a longtemps été, et est encore, conçu ${ }^{16}$.

Pour le dire en un mot, il n'y a de problème sceptique (en général, mais au sens cartésien en particulier) qu'à partir du moment où on considère que l'épistémologie devrait rendre compte de la possibilité de la connaissance humaine, prise comme un tout, de manière absolument générale et donc parfaitement a-contextuelle. Si tel est bien le but de l'entreprise, il ne semble pas y avoir d'échappatoire face à une argumentation sceptique du type du trilemme d'Agrippa: pour qu'il y ait de la connaissance, il faut qu'il y ait au moins des croyances justifiées. Mais d'où nos croyances tirent-elles leur justification? Ou bien on affirme, avec le fondationnaliste, que toute croyance tire sa justification soit d'autres croyances dont on peut la tirer inférentiellement, soit du fait qu'elle serait elle-même immédiatement justifiée, évidente ou encore indubitable. Faute de parvenir à prouver qu'il existe un tel registre de croyances privilégiées ou épistémiquement premières, le fondationnaliste tombe sous le coup de l'accusation de dogmatisme, mettant de manière fondamentalement arbitraire un terme à la régression de la chaîne des justifications. Une solution, qu'on appelle infinitisme, consisterait à soutenir que la chaîne des justifications n'a en réalité pas de fin et à épouser la régression, cependant on voit mal comment une quelconque justification pourrait logiquement être élaborée par là. L'autre solution, connue sous le nom de cohé- 
rentisme, consisterait à envisager la dynamique de la justification de manière multidimensionnelle, et non pas unidirectionnelle, ce qui conduirait à prendre comme crucial le support réciproque que différentes croyances s'assurent les unes les autres, au sein d'un système cohérent. L'objection porte alors sur le caractère circulaire d'une telle approche, qui ne permet toujours pas de comprendre d'où provient et comment se constitue la justification. Aux yeux de Michael Williams, seule une approche contextualiste peut permettre d'échapper au scepticisme, c'est-à-dire au dogmatisme comme à la circularité, pour autant qu'elle consiste à renoncer à ce qui le rend possible, et qu'il appelle le "réalisme épistémologique».

Tel que Williams le conçoit, le réalisme épistémologique correspond à la conviction que lorsque l'on parle de connaissance humaine, ou que l'on utilise les expressions "connaissance humaine » ou "croyance justifiée », on ferait par là référence à une certaine classe de propriétés que ces choses que nous considérons comme des connaissances ou des croyances justifiées partageraient, une sorte d'essence commune qui autoriserait à considérer l'ensemble des connaissances ou des croyances justifiées comme relevant d'une espèce naturelle. Or, remarque Williams, il est loin d'être évident qu'il existe une telle chose ou une telle espèce, même à titre seulement théorique. Ce que le contextualisme épistémologique permet justement de saisir, c'est que ce qui compte, pour nous, comme connaissance ou croyance justifiée, est étroitement dépendant du contexte dans lequel on évalue les propositions en question. Par exemple, on accordera à un paléoanthropologue qu'il sait, ou qu'il croit de manière justifiée que les artéfacts sur lesquels il travaille datent de l'Aurignacien, sans exiger pour cela qu'il ait d'abord prouvé qu'il existe un monde extérieur, que lui-même n'est pas un cerveau dans une cuve, ou que le monde n'a pas été créé il y a cinq minutes. Cela signifie qu'aucune proposition, prise isolément et hors de tout contexte, ne saurait avoir en elle-même de statut épistémique. Il n'y a donc pas de croyances fondamentales, qui auraient pour propriété d'être auto-justifiées ou encore «claires et distinctes ", ni de type de structure dans nos corps de croyances que l'on pourrait clairement identifier, et qui expliqueraient pourquoi certaines croyances en justifient d'autres, mais pas n'importe lesquelles. Si l'épistémologie consiste à établir quelles sortes de croyances seraient fondamentales ou bénéficieraient d'une autorité épistémique supérieure aux autres en ce senslà, alors elle est une entreprise vaine: autant, remarque Williams, essayer de trouver un point commun à toutes les choses susceptibles de se passer un mardi ${ }^{17}$. Tout comme pour nos concepts de vérité ou de réalité, l'usage de notre concept de connaissance ou de justification est soumis à de multiples contraintes, dont Williams doute fortement qu'on pourrait les réduire à un certain nombre de règles générales. La conclusion qu'il en tire, et qu'il

17. Michael Williams, «Why (Wittgensteinian) Contextualism Isn’t Relativism », Episteme, 4, 2007 , p. 98. 
semble que Jocelyn Benoist pourrait fort bien partager en l'appliquant au champ onto-sémantique, est que:

To adopt contextualism [...] is not just to hold that the epistemic status of a given proposition is liable to shift with situational, disciplinary and other contextually variable factors: it is to hold that, independently of all such influences, a proposition has no epistemic status whatsoever ${ }^{18}$.

Le fait que certaines propositions ou croyances soient tenues pour acquises, et qu'on ne les remette pas en question est une affaire de nécessité méthodologique: pour qu'une enquête, quelle qu'elle soit, soit possible, il faut que certaines présuppositions ne soient pas remises en doute. On retrouve ici les fameuses remarques de Wittgenstein dans De la Certitude:

$[\mathrm{L}]$ es questions que nous posons et nos doutes reposent sur le fait que certaines propositions sont soustraites au doute - sont, pour ainsi dire, comme des gonds sur lesquels tournent nos questions et nos doutes.

C'est-à-dire: il appartient à la logique de nos investigations scientifiques que certaines choses ne soient en fait pas mises en doute.

Mais cela ne revient pas à dire que nous ne pouvons pas tout vérifier et sommes donc obligés de nous contenter de présuppositions. Si je veux que la porte tourne, il faut que les gonds restent fixes ${ }^{19}$.

Il ne s'agit pas plus pour Williams que pour Benoist de donner un blanc seing au relativisme, notamment au relativisme épistémologique: l'objectivité de la vérité est préservée, à partir du moment du moins que l'on sépare, comme on le doit, la question du statut épistémique d'une proposition de celle de sa vérité. En effet, si le premier est bien contextuellement déterminé, ce n'est pas le cas de la seconde. Une proposition est vraie ou fausse. En revanche, on ne peut pas affirmer de la même manière, c'est-àdire hors de tout contexte ou quel que soit le contexte, qu'elle peut ou non être remise en question: "Generally speaking, a proposition is neither true because it stands fast nor stands fast because it is true ${ }^{20}$.»

Il demeure que le contextualisme qu'il défend conduit Williams à entretenir un certain scepticisme à l'égard de l'épistémologie, voire de la philosophie en général. Nous devons selon lui notamment reconnaître que s'il nous est sans doute possible de clarifier et d'expliciter notre compréhension des concepts épistémologiques, il n'est pas sûr en revanche que la «connaissance » elle-même soit quelque chose qui puisse faire l'objet d'une enquête théorique: son approche est déflationniste au sens où, encore une fois, il est très peu probable que nous puissions déterminer des «espèces

18. Michael Williams, Unnatural Doubts, p. I I9.

19. Ludwig Wittgenstein, De la certitude, traduction par Danièle Moyal-Sharrock, Paris, Gallimard, 2006 (I976), p. 98.

20. Michael Williams, Unnatural Doubts, p. I24. 
naturelles épistémologiques» susceptibles de fixer l'adresse de ce qui serait la connaissance ou la justification. Au contraire, il semble que s'il y a au moins une chose que l'épistémologie peut nous enseigner, c'est qu'une telle catégorie est vide. Après tout, on peut considérer une croyance comme justifiée, non pas parce qu'on serait capable de la justifier contre «tout le pyrrhonisme ", en toute circonstance, mais tout simplement parce, en la circonstance, il n'y a rien qui nous incite à la remettre en cause. La tâche du contextualisme épistémologique consiste précisément à identifier, à la manière d'une enquête empirique, les différents facteurs contextuels qui interviennent dans la régulation de nos pratiques épistémologiques - ce qui est très loin de la prétention qui a pu être, et est souvent encore, celle de l'épistémologie, à savoir expliquer comment la connaissance humaine est possible, en quoi elle consiste, ou encore quelles en sont les limites indépassables.

On peut se demander dans quelle mesure Jocelyn Benoist, dont le contextualisme onto-sémantique entretient plus qu'un air de famille avec celui de Williams (et pour cause: dans les deux cas, il semble que Wittgenstein soit le père!) serait disposé à tirer des conclusions du même ordre pour ce qui concerne l'ontologie et, plus largement, la philosophie elle-même. Michael Williams, volontiers pessimiste au sujet de l'épistémologie, a luimême eu l'occasion de revendiquer son appartenance, aux côtés de Quine et Rorty, à un mouvement peut-être minoritaire mais néanmoins influent durant la fin du siècle dernier, celui de la «mort de l'épistémologie ${ }^{21}$ ». Il semble s'inscrire, au répertoire de Jocelyn Benoist, une messe de requiem pour la métaphysique dont il nous a déjà donné l'introït.

21. Williams, Michael, «Death of Epistemology », in Dancy, Jonathan, Sosa, Ernst, and Steup, Matthias (eds.), A Companion to Epistemology (Second Edition), Oxford, Blackwell, 2OIO, p. 3OI-304. 